\title{
reciamuc
}

Revista cientifica de investigación actualización del mundo de las ciencias

Piedad Shirley Barragán Villafuerte a; Freddy Andrés Flores Vega ${ }^{\text {b; }}$ María

Auxiliadora Cedeño Cevallos ${ }^{c}$; Lucia Del Carmen Maza Suarez ${ }^{\mathrm{d}}$

Recanalización de la vena safena, factores de riesgo y tratamiento quirúrgico

Recanalization of the saphenous vein, risk factors and surgical treatment

Revista Científica de Investigación actualización del mundo de las Ciencias. Vol. 3 núm., 1, enero, ISSN: 2588-0748, 2018, pp. 800-831

DOI: $10.26820 /$ reciamuc/3.(1).enero.2019.800-831

URL: http://reciamuc.com/index.php/RECIAMUC/article/view/259

Código UNESCO: 3205 Medicina Interna

Tipo de Investigación: Artículo de Revisión

Editorial Saberes del Conocimiento

Recibido: 10/12/2018

Aceptado: 20/01/2019

Publicado: 30/01/2019

Correspondencia: barraganvillafuerte@gmail.com

a. Médico; Guayaquil; barraganvillafuerte@gmail.com

b. Médico; Guayaquil; aheinze77 @ hotmail.com

c. Médico Cirujano; Guayaquil; auxicede1989@ hotmail.com

d. Médico; Guayaquil; lucymedic8@hotmail.com 


\section{Recanalización de la vena safena, factores de riesgo y tratamiento quirúrgico}

Vol. 3, núm. 1., (2019)

Piedad Shirley Barragán Villafuerte; Freddy Andrés Flores Vega; María Auxiliadora Cedeño

Cevallos; Lucia Del Carmen Maza Suarez

\section{RESUMEN}

La enfermedad venosa crónica constituye una de las enfermedades más prevalentes en los humanos y es el principal motivo de consulta por patología vascular en asistencia primaria. En países latinoamericanos, las enfermedades vasculares representan un 3,4\% de las consultas, de las cuales el $69 \%$ corresponden a enfermedad venosa crónica. Esta patología tiene una elevada morbilidad en estadios avanzados, por lo que su prevención es muy importante permitiendo por otra parteracionalizar los recursos necesarios para su control. La misión del cirujano vascular es la de valorar y tratar los pacientes muy sintomáticos, con complicaciones establecidas, o que requieran diagnóstico y tratamiento especializado, sea endo - vascular o quirúrgico. Las venas varicosas afectan hasta el $40 \%$ de la población adulta, causando síntomas que perturban la calidad de vida. Las manifestaciones más comunes resultan del reflujo en la vena safena interna (VSI). Existen muchos métodos para tratar el reflujo en la VSI: cirugía abierta, escleroterapia con espuma (EE), y ablación con catéter endovenoso utilizando, por ejemplo, radiofrecuencia o energía láser. El proceso de recanalización del trombo, se realiza a través de un proceso que produce la destrucción del endotelio valvular de los segmentos implicados, de forma que la hipertensión venosa se estará desarrollando en relación al reflujo provocado, solo o combinado con un mayor o menor grado de obstrucción del drenaje venoso. La hipertensión en este caso, se encuentra asociada con la inflamación crónica lo que afecta, no sólo a la pared venosa, sino también a la microcirculación, dando lugar a las alteraciones tróficas cutáneas. De allí, que la prevención del síndrome post-trombótico se basa en la correcta dosificación y duración del tratamiento anticoagulante tras una trombosis venosa profunda, junto con la movilización precoz y la inmediata compresión elástica del miembro afecto.

Palabras Claves: Vena Safena; Factores de Riesgo; Tratamiento Quirúrgico; Enfermedades Vasculares; Recanalización del Trombo. 


\title{
Recanalización de la vena safena, factores de riesgo y tratamiento quirúrgico
}

Vol. 3, núm. 1., (2019)

Piedad Shirley Barragán Villafuerte; Freddy Andrés Flores Vega; María Auxiliadora Cedeño Cevallos; Lucia Del Carmen Maza Suarez

\begin{abstract}
Chronic venous disease is one of the most prevalent diseases in humans and is the main reason for consultation for vascular pathology in primary care. In Latin American countries, vascular diseases account for $3.4 \%$ of consultations, of which $69 \%$ correspond to chronic venous disease. This pathology has a high morbidity in advanced stages, so its prevention is very important allowing on the other hand to rationalize the necessary resources for its control. The mission of the vascular surgeon is to assess and treat very symptomatic patients, with established complications, or who require diagnosis and specialized treatment, whether endovascular or surgical. Varicose veins affect up to $40 \%$ of the adult population, causing symptoms that disrupt quality of life. The most common manifestations result from reflux in the internal saphenous vein (VSI). There are many methods to treat reflux in the VSI: open surgery, foam sclerotherapy (EE), and endovenous catheter ablation using, for example, radiofrequency or laser energy. The process of recanalization of the thrombus is carried out through a process that produces the destruction of the valvular endothelium of the involved segments, so that the venous hypertension will be developing in relation to the provoked reflux, alone or combined with a greater or lesser degree of obstruction of the venous drainage. In this case, hypertension is associated with chronic inflammation, which affects not only the venous wall, but also the microcirculation, leading to cutaneous trophic alterations. Hence, the prevention of postthrombotic syndrome is based on the correct dosage and duration of anticoagulant treatment after deep vein thrombosis, together with early mobilization and immediate elastic compression of the affected limb.
\end{abstract}

Key Words: Safena vein; Risk factor's; Surgical Treatment; Vascular diseases; Recanalization of the Trombo. 


\section{Recanalización de la vena safena, factores de riesgo y tratamiento quirúrgico}

Vol. 3, núm. 1., (2019)

Piedad Shirley Barragán Villafuerte; Freddy Andrés Flores Vega; María Auxiliadora Cedeño Cevallos; Lucia Del Carmen Maza Suarez

\section{Introducción.}

La enfermedad venosa crónica (EVC) constituye una patología conocida desde la antigüedad que afecta a un 25\% de la población adulta occidental. En el año 2009 se consensuó su definición como cualquier anormalidad morfológica y funcional del sistema venoso de larga evolución, manifestada mediante síntomas que requieren de investigación, o atención médica. La EVC, y su signo más paradigmático, la presencia de varices, afectan a más del $60 \%$ de la población en países latinoamericanos.

Las alteraciones en las válvulas, o pared de la vena representan el desencadenante de la hipertensión venosa, hecho fisiopatológico fundamental que va a ser el responsable de las manifestacionesclínicas de la enfermedad: dolor, pesadez, calambres, prurito, varices, úlceras y trastornos dérmicos entre otros. (Quetz, 2016). La EVC es de etiología primaria en el 95\% de casos y soloun $4-5 \%$ es secundaria, sobre todo por trombosis venosa profunda. Las angio displasias, enfermedades neuromusculares que afectan a las bombas musculares o compresiones extrínsecas constituyen otras causas menos frecuentes.

Las venas varicosas son frecuentes en muchas poblaciones, con tasas de prevalencia que, en general, varían desde el 10,4 al $23,0 \%$ en hombres y del 29,5 al $39,0 \%$ en mujeres: En estadísticas arrojadas de estudios realizados por la Organización Mundial de la Salud OMS 2016, se encontró que la incidencia bianual de venas varicosas es del $2 \%$ en hombres y el $3 \%$ en mujeres En mismo estudio se sugirió que, durante un período de 2 años más, el 2,0\% de los hombres y el 2,6\% de las mujeres desarrollarían venas varicosas. Según (Palett, 2017): 


\section{Recanalización de la vena safena, factores de riesgo y tratamiento quirúrgico}

Vol. 3, núm. 1., (2019)

Piedad Shirley Barragán Villafuerte; Freddy Andrés Flores Vega; María Auxiliadora Cedeño Cevallos; Lucia Del Carmen Maza Suarez

La idoneidad del paciente para el tratamiento de las venas varicosas se establece a través de la exploración clínica, la cual permitirá determinar el origen de la incompetencia venosa y el tipo y extensión de las venas que han de tratarse. En condiciones ideales, este examen va seguido de un eco-Doppler para confirmar la presencia de reflujo. La enfermedad venosa se define usando una serie de sistemas de clasificación, ninguno de los cuales se acepta de forma universal. (p.12).

De aquí, la importancia de brindarle la debida atención al procedimiento terapéutico elegido por el especialista tratante de venas varicosas, el cual debe ir acompañado junto a un equipamiento adecuado, teniendo siempre la formación y capacitación necesaria para discernir entre una u otra técnica a desarrollar para su tratamiento y de las posibilidades terapéuticas que el mismo brinda, considerando las implicaciones que comprende el tema de la insuficiencia venosa. Al respecto (Génova, 2016), plantea:

La insuficiencia venosa se define como la incapacidad del sistema venoso para mantener una presión intraluminal capilar inferior a la presión coloidosmótica, lo que impide la adecuada función hemodinámica de reabsorción capilar. La misma, se caracteriza por la hipertensión venosa y sus consecuencias fisiopatológicas, siendo más frecuente en miembros inferiores, ya que en ellos influye negativamente la presión hidrostática. (p.14).

Lo antes descrito lleva a considerar que, la IVC representa una alteración de la función venosa normal, lo cual se debe a la insuficiencia valvular u obstrucción al flujo, que puede afectar a cualquier segmento venoso y ser primaria o secundaria a trombosis previa, generando o 


\section{Recanalización de la vena safena, factores de riesgo y tratamiento quirúrgico}

Vol. 3, núm. 1., (2019)

Piedad Shirley Barragán Villafuerte; Freddy Andrés Flores Vega; María Auxiliadora Cedeño Cevallos; Lucia Del Carmen Maza Suarez

produciendo el aumento de la presión venosa. Esta presencia de las varices, conduce a un cuadro evolutivo, en el cual pueden ir sobreviniendo distintas complicaciones que van agravando el cuadro, limitando la calidad de vida y la salud del paciente.

En efecto, la insuficiencia venosa de miembros inferiores, es uno de los trastornos vasculares más frecuentes de la población. No se conoce con precisión la etiopatogenia, no obstante existen factores predisponentes evidentes: siendo más frecuente en la mujer que en el varón, lo que va acompañado de estadísticas donde se refleja que existen antecedentes familiares en el 50\% de los casos, predominan en la raza blanca. Del mismo modo, (Blom, 2016), señala:

La vena safena interna recorre la cara interna del miembro inferior, originándose en la región infra maleolar interna y formándose de la reunión de las venas marginales mediales que proceden de la región interna del dorso del pie y de la región plantar interna. Esta vena, asciende en posición intraaponeurótica desde el tobillo (en posición premaleolar) por la cara interna de la pierna, cara interna gonal y cara interna del muslo, hasta desembocar en forma de cayado en la vena femoral común, en el ostium safeno-femoral. (p.13).

Entiéndase con esto que, la vena Safena Interna (SI), recibe en ocasiones a la vena Safena Anterior (SA) la cual puede conformar un tronco común con la safena interna, que es lo más frecuente o desembocar en la vena femoral común (VFC) de forma independiente, y en ocasiones en estrecha relación con la arteria pudenda externa que cruza el cayado por su cara anterior.Esta vena, atraviesa la ventana oval aponeurótica en forma de cayado, uniéndose a la vena femoral común (VFC) conformando el ostium safeno-femoral (S-F). 


\section{Recanalización de la vena safena, factores de riesgo y tratamiento quirúrgico}

Vol. 3, núm. 1., (2019)

Piedad Shirley Barragán Villafuerte; Freddy Andrés Flores Vega; María Auxiliadora Cedeño Cevallos; Lucia Del Carmen Maza Suarez

La anatomía clásica de esta vena, describe tres colaterales típicas: Subcutánea abdominal o epigástrica. Vn. Pudenda externa (PUD), en posición medial Y Circunfleja iliaca externa (CIE), en posición lateral.Las venas safena externa (VSE) y safena interna (VSI) forman parte del sistema venoso superficial. La mayor parte de la sangre de las piernas regresa al corazón, a través de las venas que forman parte del sistema venoso profundo; por lo tanto, la sangre que previamente ha recorrido el territorio de la vena safena puede redirigirse a través del sistema venoso profundo en caso de distensión o varicosidades de la VSI.

En pacientes con venas de mayor calibre o reflujo venoso son habituales elevadas tasas de recidiva' principalmente debido a la recanalización o neovascularización. Entiéndase por recanalización al restablecimiento espontáneo de la luz de la vena safena tras la oclusión, mientras que la neovascularización es la proliferación de los vasos sanguíneos en el tejido donde previamente se ha extirpado la vena safena.

\section{Tipo de Investigación}

Dentro de toda práctica investigativa, se precisan acciones de carácter metodológico mediante las cuales, se logra conocer y proyectar los eventos posibles que la determinan, así como las características que hacen del acto científico un proceso interactivo ajustado a una realidad posible de ser interpretada. En este sentido, se puede decir, que la presente investigación corresponde al tipo documental, definido por (Dávila, 2012), "se ocupa del estudio de problemas planteados a nivel teórico, la información requerida para abordarlos se encuentra básicamente en materiales impresos, audiovisuales y /o electrónicos”. (p.41). 


\section{Recanalización de la vena safena, factores de riesgo y tratamiento quirúrgico}

Vol. 3, núm. 1., (2019)

Piedad Shirley Barragán Villafuerte; Freddy Andrés Flores Vega; María Auxiliadora Cedeño Cevallos; Lucia Del Carmen Maza Suarez

En consideración a esta definición, la orientación metodológicapermitió la oportunidad de cumplir con una serie de actividades inherentes a la revisión y lectura de diversos documentos donde se encontraron ideas explicitas relacionadas con los tópicos encargados de identificar a cada característica insertada en el estudio. Por lo tanto, se realizaron continuas interpretaciones con el claro propósito de revisar aquellas apreciaciones o investigaciones propuestas por diferentes investigadores, para luego dar la respectiva argumentación a los planteamientos, en función a las necesidades encontradas en la indagación.

\section{Fuentes Documentales}

El análisis correspondiente a las características que predomina en el tema seleccionado, llevan a incluir diferentes fuentes documentales encargadas de darle el respectivo apoyo y en ese sentido cumplir con la valoración de los hechos a fin de generar nuevos criterios que sirven de referencia a otros procesos investigativos. Para (Arias, 2010), las fuentes documentales incorporadas en la investigación documental o bibliográfica, "representa la suma de materiales sistemáticos que son revisados en forma rigurosa y profunda para llegar a un análisis del fenómeno". (p.41). Por lo tanto, se procedió a cumplir con la realización de una lectura previa determinada por encontrar aquellos aspectos estrechamente vinculados con la "Recanalización de la vena safena, factores de riesgo y tratamiento Quirúrgico”, para luego explicar mediante un desarrollo las respectivas apreciaciones generales de importancia.

\section{Técnicas para la Recolección de la Información}

La conducción de la investigación para ser realizada en función a las particularidades que determinan a los estudios documentales, tiene como fin el desarrollo de un conjunto de acciones 


\section{Recanalización de la vena safena, factores de riesgo y tratamiento quirúrgico}

Vol. 3, núm. 1., (2019)

Piedad Shirley Barragán Villafuerte; Freddy Andrés Flores Vega; María Auxiliadora Cedeño Cevallos; Lucia Del Carmen Maza Suarez

encargadas de llevar a la selección de técnicas estrechamente vinculadas con las características del estudio. En tal sentido, (Arias Obcit) refiere, que es "una técnica particular para aportar ayuda a los procedimientos de selección de las ideas primarias y secundarias”. (p. 71).

Por ello, se procedió a la utilización del subrayado, resúmenes, fichaje, como parte básica para la revisión y selección de los documentos que presentan el contenido teórico. Es decir, que mediante su aplicación de estas técnicas se pudo llegar a recoger informaciones en cuanto a la revisión bibliográfica de los diversos elementos encargados de orientar el proceso de investigación. Tal como lo expresa, (Arias Obcit) "las técnicas documentales proporcionan las herramientas esenciales y determinantes para responder a los objetivos formulados y llegar a resultados efectivos" (p. 58). Es decir, para responder con eficiencia a las necesidades investigativas, se introdujeron como técnica de recolección el método inductivo, que hizo posible llevar a cabo una valoración de los hechos de forma particular para llegar a la explicación desde una visión general.

Asimismo, se emplearon las técnicas de análisis de información para la realización de la investigación que fue ejecutada bajo la dinámica de aplicar diversos elementos encargados de determinar el camino a recorrer por el estudio, según, (Arias, Obcit) las técnicas de procesamiento de datos en los estudios documentales "son las encargadas de ofrecer al investigador la visión o pasos que debe cumplir durante su ejercicio, cada una de ellas debe estar en correspondencia con el nivel a emplear" (p. 123). Esto indica, que para llevar a cabo el procesamiento de los datos obtenidos,es necesario establecer las técnicas que serán seleccionadas, destacándose en este caso, de manera particular: fichas de resumen, textual, registros descriptivos entre otros, los mismos se deben ajustar al nivel que ha sido seleccionado. 


\section{Recanalización de la vena safena, factores de riesgo y tratamiento quirúrgico}

Vol. 3, núm. 1., (2019)

Piedad Shirley Barragán Villafuerte; Freddy Andrés Flores Vega; María Auxiliadora Cedeño Cevallos; Lucia Del Carmen Maza Suarez

\section{Resultados.}

Vena Safena

La vena safena es continuación de la vena dorsal medial y, por medio de esta última, de la extremidad medial del arco dorsal del pie. Verticalmente ascendente, escoltado por el nervio safeno, pasa por delante del maléolo tibial, luego la cara medial de la pierna y de la rodilla y la cara antero medial del muslo, hasta llegar a 3 o 4 centímetros debajo del ligamento inguinal. En todo su recorrido la vena es superficial hasta llegar a su cayado en donde se vuelve profunda. Es muy rica en válvulas, muy variables en su situación, desarrollo y número. Mientras más joven el individuo, mayor el número de válvulas; se cuentan 11 a 20 en el adulto y hasta 30 en un feto. Algunas son insuficientes y están destinadas a desaparecer. Se encuentran cerca de las articulaciones de la rodilla y el tobillo; existe siempre constante una válvula en la proximidad del cayado.

Várices de la safena magna

Las patologías de la vena safena magna son relativamente frecuentes y aisladamente son enfermedades benignas:

- Enfermedad varicosa del miembro inferior: La safena magna, así como otras venas superficiales, se pueden volver varicosas (hinchadas, tortuosas y alargadas que pueden producir dolor). 


\section{Recanalización de la vena safena, factores de riesgo y tratamiento quirúrgico}

Vol. 3, núm. 1., (2019)

Piedad Shirley Barragán Villafuerte; Freddy Andrés Flores Vega; María Auxiliadora Cedeño Cevallos; Lucia Del Carmen Maza Suarez

- Tromboflebitis: Es una inflamación de las venas con riesgo a formar trombos. La safena magna es propensa a trombosarse cuando es varicosa; se vuelve peligroso cuando se asocia a trombosis venosa profunda.

La safenectomía es un proceso quirúrgico en donde se liga y extirpa la vena safena magna. Se utiliza con el propósito de tratar la enfermedad varicosa. También se usa este procedimiento en el autotrasplante para la revascularización, uno de los procedimientos más realizados en cirugía cardíaca.Se puede utilizar como vía de acceso parenteral en administración de fármacos endovenosos o en nutrición parenteral en pacientes pediátricos.(Angiología, 2018).

\section{Extirpación de venas varicosas}

La extirpación venosa o fleboextracción es la cirugía para extirpar venas varicosas de las piernas.

\section{Descripción.}

Las venas varicosas son venas hinchadas, retorcidas y dilatadas que se pueden ver bajo la piel. A menudo son de color rojo o azul. Por lo general, aparecen en las piernas, pero se pueden presentar en otras partes del cuerpo. Normalmente, las válvulas en las venas mantienen la sangre circulando hacia el corazón, de manera que no se acumule en un lugar. En las venas varicosas, las válvulas están dañadas o ausentes. Esto provoca que las venas resulten llenas de sangre, especialmente cuando uno está de pie. 


\section{Recanalización de la vena safena, factores de riesgo y tratamiento quirúrgico}

Vol. 3, núm. 1., (2019)

Piedad Shirley Barragán Villafuerte; Freddy Andrés Flores Vega; María Auxiliadora Cedeño Cevallos; Lucia Del Carmen Maza Suarez

La extirpación venosa se utiliza para extraer o ligar una vena grande en la pierna llamada vena safena superficial. Esto ayuda a tratar las venas varicosas. Este procedimiento, por lo general tarda de 1 a $1 \frac{1}{2}$ horas aproximadamente, ya sea, a través de:

- Anestesia general, con la cual el (la) paciente estará dormido (a) y no podrá sentir dolor.

- Anestesia raquídea, la cual insensibilizará la mitad inferior del cuerpo. A usted también le pueden dar un medicamento para ayudar a que se relaje.

Durante la cirugía:

- El cirujano hará 2 o 3 cortes quirúrgicos pequeños en la pierna.

- Los cortes estarán cerca de la parte superior, media e inferior de la vena dañada. Uno estará en la ingle. El otro estará más abajo en la pierna, ya sea en la pantorrilla o en el tobillo.

- El cirujano pasará luego un cable plástico flexible y delgado hasta la vena a través de la ingle y lo guiará a través de la vena hacia el otro corte más abajo en la pierna.

- Luego, se ata el cable a la vena y se hala hacia afuera a través del corte inferior, con lo cual se extrae la vena.

- Si usted tiene otras venas dañadas cerca de la superficie de la piel, el cirujano también puede hacer incisiones pequeñas sobre ellas para extirparlas o ligarlas. Esto se denomina flebectomía ambulatoria.

- El cirujano cerrará las incisiones con suturas (puntos de sutura).

- Usted usará vendajes y medias de compresión en la pierna después del procedimiento. 


\section{Recanalización de la vena safena, factores de riesgo y tratamiento quirúrgico}

Vol. 3, núm. 1., (2019)

Piedad Shirley Barragán Villafuerte; Freddy Andrés Flores Vega; María Auxiliadora Cedeño Cevallos; Lucia Del Carmen Maza Suarez

Tabla $N^{\circ} 1$. Tratamiento quirúrgico de la insuficiencia venosa en el territorio de la vena safena

externa

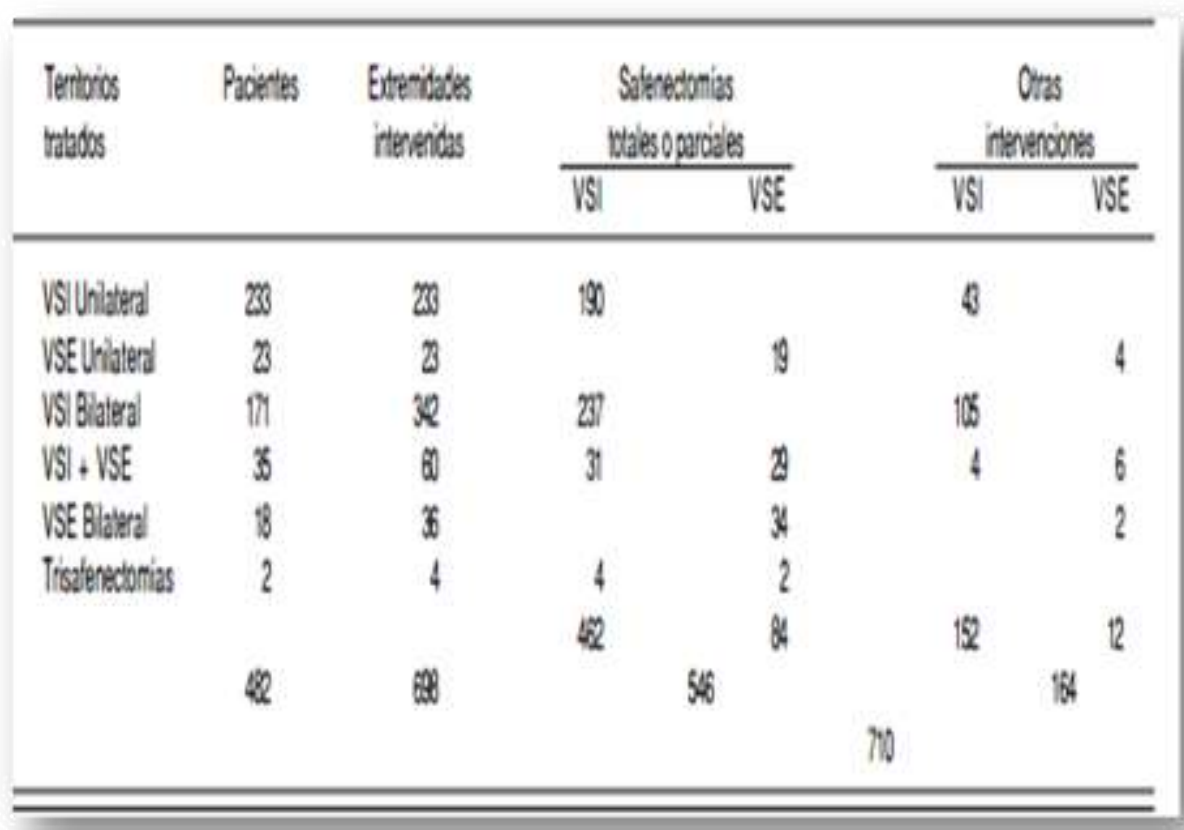

Fuente: Revista Mexicana de Angiología (2018)

El procedimiento desafenectomía clásica sigue siendo una técnica útil, barata y con excelentes resultados en el tratamiento de las várices dependientes de la VSE. La morbilidad neurológica precoz, pese a todas las medidas implementadas para controlarla, es la complicación más frecuente y molesta de la denudación venosa. Los costos económicos de esta cirugía son muy bajos, no obstante, debe mejorarse el seguimiento de la incapacidad temporal tras la intervención, ya que se han detectado bajas prolongadas que no se justifican desde el punto de vista clínico. 


\section{Recanalización de la vena safena, factores de riesgo y tratamiento quirúrgico}

Vol. 3, núm. 1., (2019)

Piedad Shirley Barragán Villafuerte; Freddy Andrés Flores Vega; María Auxiliadora Cedeño Cevallos; Lucia Del Carmen Maza Suarez

Fisiopatología de la Enfermedad Venosa Crónica ECV

La patogénesis de la EVC representa un proceso complejo y probablemente multifactorial.El retorno de la sangre venosa desde las extremidades inferiores al corazón se realiza mediante dos circuitos en paralelo: el sistema venoso profundo (SVP), localizado dentrode la fascia profunda que envuelve a los músculos profundos de las extremidades inferiores, y el sistema venoso superficial (SVS). Ambos se encuentran interconectados por un tercer sistema, el de las venas perforantes. A grandes rasgos, el SVS se inicia en las venas del pie para formar la vena safena mayor (interna) y la safena menor (externa), que desembocan en la vena femoral común a nivel del muslo y en la vena poplítea, respectivamente.

Figura $N^{\circ} 1$. Vena Safena menor o externa (A) y mayor o interna (B). Sistema venoso superficial, profundo y venas perforantes, las flechas indican la dirección del flujo (C)

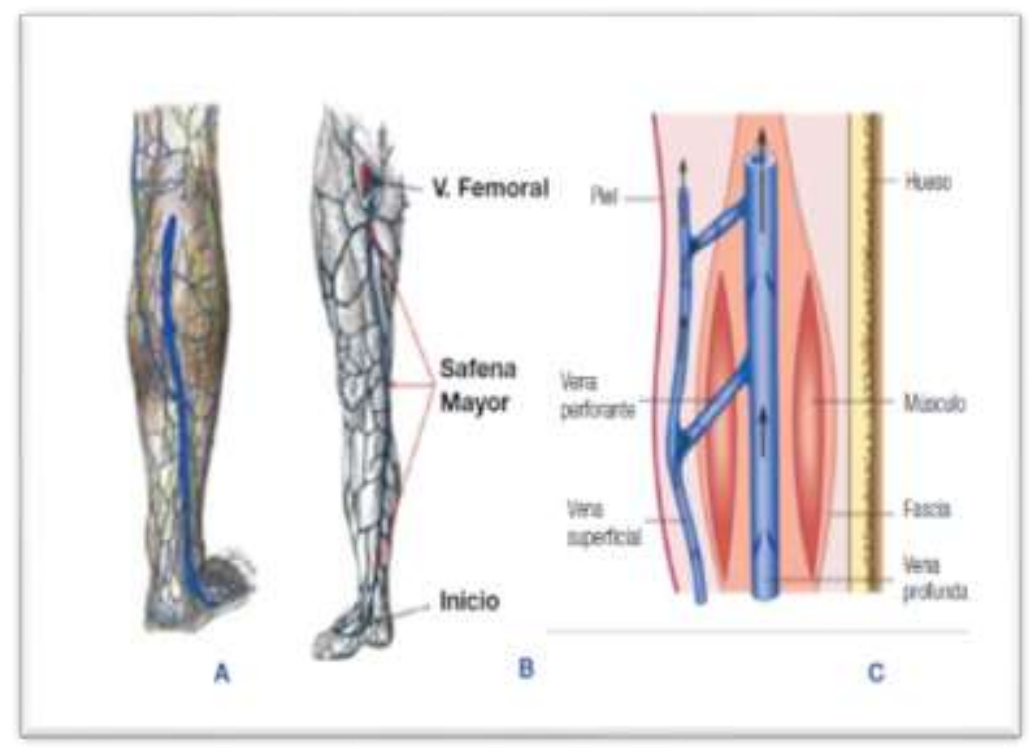

Fuente: Vayaretti (2016) 


\section{Recanalización de la vena safena, factores de riesgo y tratamiento quirúrgico}

Vol. 3, núm. 1., (2019)

Piedad Shirley Barragán Villafuerte; Freddy Andrés Flores Vega; María Auxiliadora Cedeño Cevallos; Lucia Del Carmen Maza Suarez

En bipedestación, el retorno venoso se hace contra gravedad y se favorece por la presenciade válvulas venosas que impiden el flujo retrógrado, por la acción de bombeo que ejercen los músculos gemelares y del pie durante la marcha, por la diástole cardiaca ylos movimientos respiratorios. El flujo venoso siempre circula en el mismo sentido, de lasvenas superficiales a las profundas y de ahí al corazón.(Vayaretti, 2016).

Asimismo, la columna de sangre venosa entre la aurícula derecha y el pie ejerce una presión hidrostática en el tobillo de unos 90-100 mmHg en bipedestación, lo que dificulta el flujo venoso normal hacia el corazón. Los mecanismos valvulares y las bombas musculares son capaces de desarrollar una contrapresión que vacía las venas profundas, haciendo disminuir la presión a menos de $25 \mathrm{mmHg}$. Esta presión se conoce como presión venosa ambulatoria (PVA). En contraste, la presión en el SVS es muy baja, solo de5-10 mmHg en posición supina. Durante la relajación muscular, la sangre fluye desde elsistema venoso superficial al SVP.Vayaretti (Obcit) 
Recanalización de la vena safena, factores de riesgo y tratamiento quirúrgico

Vol. 3, núm. 1., (2019)

Piedad Shirley Barragán Villafuerte; Freddy Andrés Flores Vega; María Auxiliadora Cedeño Cevallos; Lucia Del Carmen Maza Suarez

Figura $N^{\circ}$ 2. Retorno venoso en bipedestación por acción de la bomba plantar y gemelar. A, B: la bomba plantar impulsa la sangre hacia la pantorrilla. C: la contracción gemelar vuelve a impulsar la sangre hacia el corazón

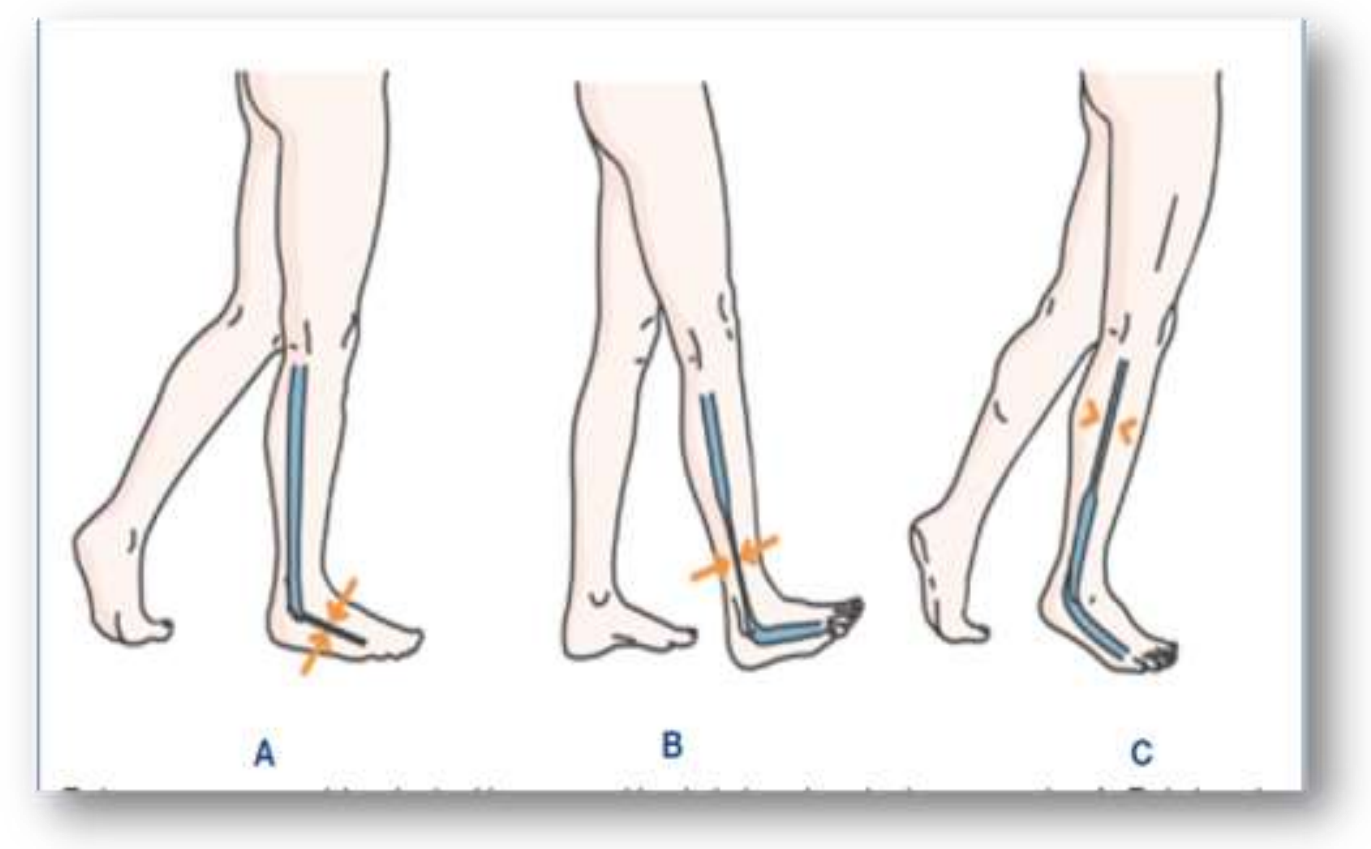

Fuente: Vayaretti (2016).

El factor determinante de la aparición de la EVC primaria es la incompetencia de las válvulas venosas por una alteración de las mismas o por un defecto idiopático estructural de la pared venosa, o por ambos mecanismos; lo que provoca dilatación venosa con fallos en el cierre de sus válvulas. La EVC puede ser secundaria a una trombosis venosa profunda que provoca destrucción valvular u obstrucción al flujo. En otras ocasiones las alteraciones se producen por compresión extrínseca o malformaciones. La incompetencia valvular genera un reflujo venoso que provoca hipertensiónvenosa (HTV), condicionante esta de la dilatación de las venas del 


\section{Recanalización de la vena safena, factores de riesgo y tratamiento quirúrgico}

Vol. 3, núm. 1., (2019)

Piedad Shirley Barragán Villafuerte; Freddy Andrés Flores Vega; María Auxiliadora Cedeño Cevallos; Lucia Del Carmen Maza Suarez

SVS(varices), de las alteraciones en la microcirculación y lesiones tróficas, responsables a su vez de las manifestaciones clínicas.

Los síntomas de ECV, pueden acompañar a todas las etapas. El dolor en la enfermedad venosa es la queja principal que conduce al diagnóstico de EVC. Las hipótesis actuales sobre los mecanismos del dolor venoso se centran en un origen inflamatorio local. Se ha postulado que los mediadores pro-inflamatorios liberados localmente por los leucocitos pueden activar fibras amielínicas situadas en la pared venosa como una amplia malla que surge de la adventicia extendiéndose por la parte externa de la media y en el tejido conectivo que forma el espacio peri venoso, en estrecho contacto con la microcirculación. El estímulo de estas fibras produce un dolor difuso y probablemente también síntomas neuro - paticos como pesadez, parestesias, etc.

\section{Epidemiología de la Enfermedad Venosa Crónica ECV}

Existe numerosa bibliografía que informa del alto impacto sanitario (elevada prevalencia y las consecuencias asistenciales), social (alteración de la calidad de vida y costes laborales), económico (gastos directos, indirectos e intangibles) de la EVC, constituyendo un importante problema de salud pública. La EVC es la enfermedad vascular más frecuente del ser humano. Esta enfermedad, es más frecuente en mujeres que en hombres. El 25-30\% de mujeres y 10-40\% de hombres occidentales tienen varices. (Colling, 2016). 


\section{Recanalización de la vena safena, factores de riesgo y tratamiento quirúrgico}

Vol. 3, núm. 1., (2019)

Piedad Shirley Barragán Villafuerte; Freddy Andrés Flores Vega; María Auxiliadora Cedeño Cevallos; Lucia Del Carmen Maza Suarez

\section{Tabla $N^{\circ}$ 2. Estudios Españoles de prevalencia de EVC}

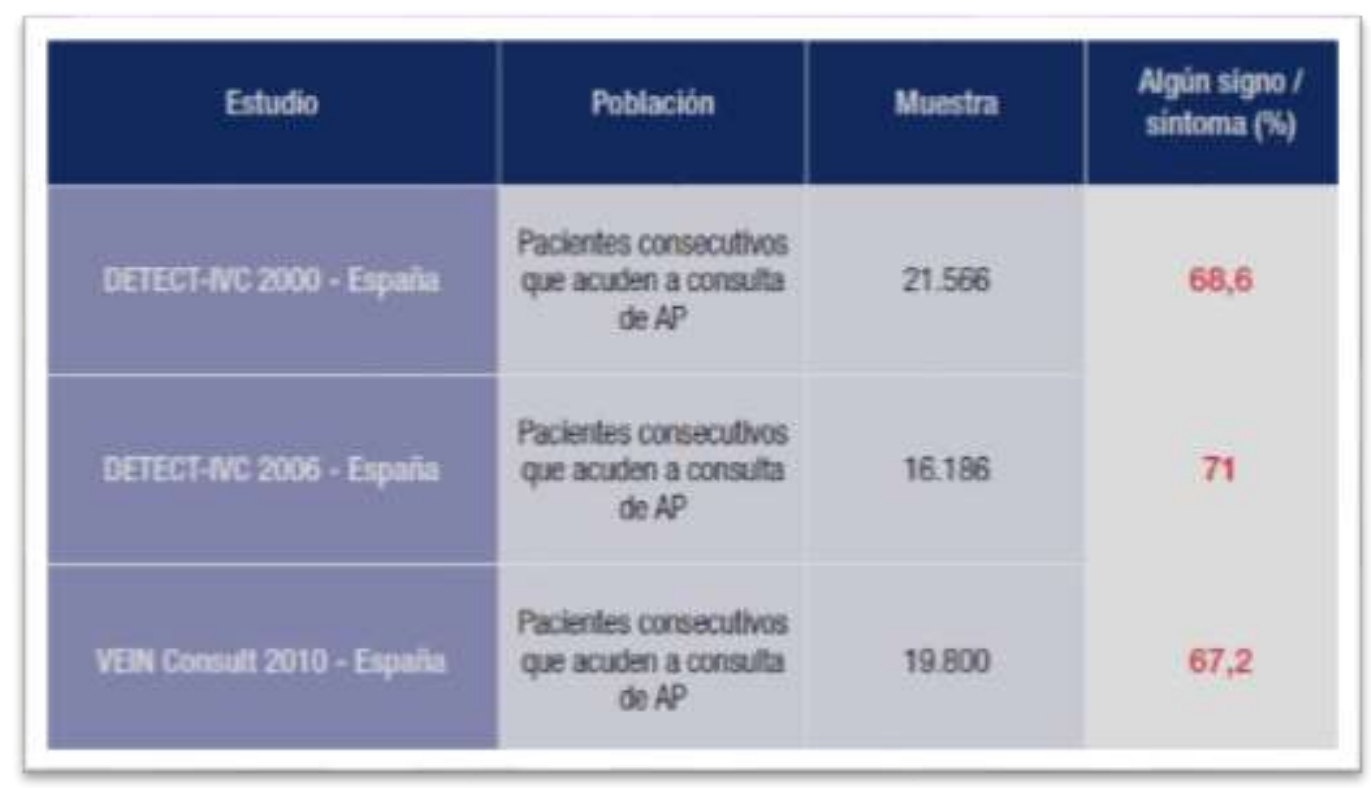

Fuente: Revista de Anatomía Venosa de España (2017)

La incidencia anual de varices, según el estudio Framingham, está en el 2,6\% para las mujeres y en el 1,9\% para los hombres. La prevalencia de la EVC aumenta linealmente con la edad pero puede aparecer en niños y adolescentes. El 77\% de mujeres mayores de 70 años tienen varices. El pico de incidencia de la EVC ocurre a los 40-49 años en mujeres y 70-79 años en hombres.(Queralt, 2017)

Factores de riesgo

Para realizar un diagnóstico adecuado de la ECV, se necesita tener conocimiento de los factores de riesgo de la EVC, a fin de lograr con ello planearactuaciones e intervenciones que 


\section{Recanalización de la vena safena, factores de riesgo y tratamiento quirúrgico}

Vol. 3, núm. 1., (2019)

Piedad Shirley Barragán Villafuerte; Freddy Andrés Flores Vega; María Auxiliadora Cedeño Cevallos; Lucia Del Carmen Maza Suarez

retrasen o impidan el comienzo de la enfermedad. Dentro de los factores predisponentes para la EVC se reconocen los siguientes:

- Genéticos: Parece que existe una predisposición genética. La EVC se transmite de forma variable, unas veces con carácter recesivo y otras dominantes. Algunos trabajos muestran que el riesgo de que los hijos desarrollen venas varicosas es del $89 \%$ si ambos padres sufren EVC, del $47 \%$ si solo un progenitor la sufre y del $20 \%$ si ninguno de ellos tiene evidencia de EVC.

- Edad: es el principal factor de riesgo. Con la edad se producen cambios estructurales en la pared venosa que facilitan su dilatación. Es un factor de riesgo independiente.

- Sexo: es el segundo factor en importancia. Predomina en el sexo femenino a razón de 2-8:1 con respecto al hombre, aunque en estudios poblacionales no hay diferencia de sexo, presentando varices con la misma frecuencia. Sin embargo, hay una mayor incidencia de varices reticulares y telangiectasias en la mujer, mientras que en el hombre predominan las tronculares y estadios más avanzados de enfermedad.

- Peso: se ha demostrado repetidamente en los estudios poblacionales una mayor incidencia de EVC en obesos o con sobrepeso importante. 


\section{Recanalización de la vena safena, factores de riesgo y tratamiento quirúrgico}

Vol. 3, núm. 1., (2019)

Piedad Shirley Barragán Villafuerte; Freddy Andrés Flores Vega; María Auxiliadora Cedeño Cevallos; Lucia Del Carmen Maza Suarez

\section{Tabla $N^{\circ}$ 3. Etiología y factores de riesgo de la ECV}

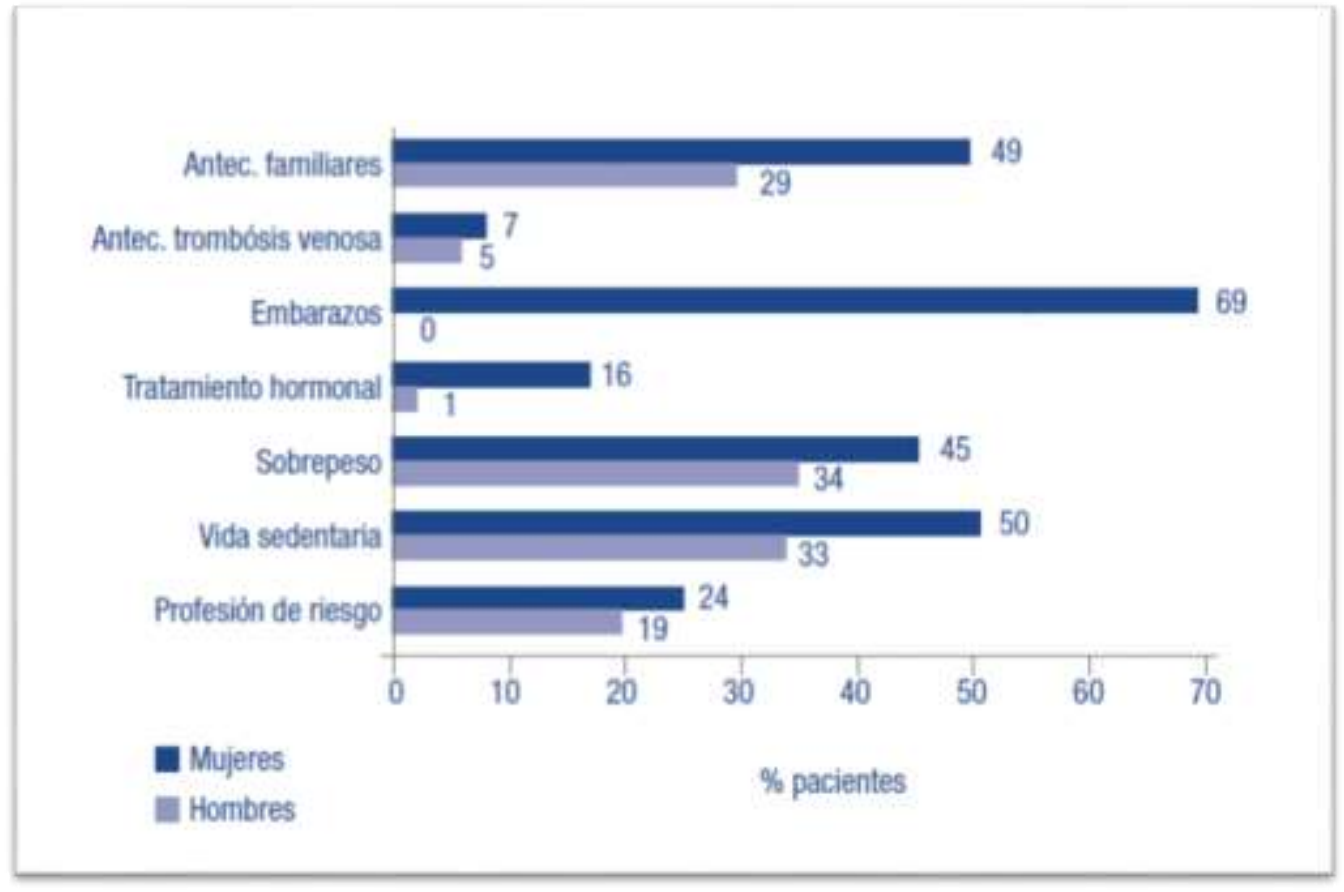

Fuente: Revista de Anatomía Venosa de España (2017)

- Gestación: la EVC es más frecuente en mujeres multíparas. El embarazo favorecería la aparición de EVC a través de 3 mecanismos: los cambios hormonales que tienen lugar durante el embarazo que provocan disminución del tono venoso, el incremento de la volemia y el aumento de la presión intraabdominal que altera el retorno venoso.

- Raza: es más frecuente en raza blanca y muy poco común en raza negra y asiática. Se ha descrito una mayor frecuencia de aparición de EVC en países nórdicos y centroeuropeos que en países mediterráneos.

- Hábitos intestinales: el estreñimiento favorece la aparición de varices por un aumento de la presión intraabdominal. 


\section{Recanalización de la vena safena, factores de riesgo y tratamiento quirúrgico}

Vol. 3, núm. 1., (2019)

Piedad Shirley Barragán Villafuerte; Freddy Andrés Flores Vega; María Auxiliadora Cedeño Cevallos; Lucia Del Carmen Maza Suarez

- Exposición al calor: puede producir una venodilatación cutánea y enlentecimiento circulatorio.

- Compresiones: locales o selectivas (prendas de vestir ajustadas, vendajes, cinturones, medias, zapatos...).

- Ocupación laboral: aumenta su incidencia en trabajos con bipedestación prolongada.

- Tratamiento hormonal: La toma de anticonceptivos orales se ha relacionado con la aparición de EVC. Altas dosis de progesterona aumentan ladistensibilidad venosa y altas dosis de estrógenos aumentan la hipercoagulabilidad.

Asimismo, se observó que en el estudio español DETECT 2006, los factores de riesgo más prevalentes en mujeresfueron, por orden: el embarazo, vida sedentaria, antecedentes familiares, sobrepeso y las profesiones de riesgo (aquellas en las que una persona pasa más de 5 horas de su jornada laboral de pie o sentado: peluqueros, camareros, recepcionistas, etc.); mientras que en los hombres fueron: el sobrepeso, vida sedentaria, antecedentes familiares y las profesiones de riesgo.

\section{Clínica de la Enfermedad Venosa Crónica ECV}

La presentación clínica de la enfermedad venosa crónica (EVC) es variada y cambiante. Su evolución, va desde problemas estéticos hasta la presencia de signos graves, entre ellos la aparición de úlceras. Los síntomas, aunque son inespecíficos, orientan hacia su presencia por lo que es necesario su conocimiento al igual que los trastornos dérmicos asociados. 


\section{Recanalización de la vena safena, factores de riesgo y tratamiento quirúrgico}

Vol. 3, núm. 1., (2019)

Piedad Shirley Barragán Villafuerte; Freddy Andrés Flores Vega; María Auxiliadora Cedeño Cevallos; Lucia Del Carmen Maza Suarez

Dolor

Parece estar ocasionado por la liberación de mediadores inflamatorios, consecutivaa la hipertensión venosa mantenida que estimulan las fibras nociceptoras subendoteliales. Suele ser de carácter difuso, no localizado, subagudo o crónico. Se exacerba con tratamientos hormonales (anticonceptivos), sedentarismo, bipedestación prolongada o altas temperaturas.

- Los dolores tardíos son más frecuentes, se intensifican durante el transcurso del día, aliviándose con el ejercicio, el frío o la deambulación.

- Los dolores de tipo inmediato suelen aparecer al levantarse, como una sensación de llenado que recorre el miembro.

- También es frecuente el dolor en el decúbito, con sensaciones de piernas no descansadas o inquietas. Los dolores puntuales, generalmente agudos, suelen ser manifestaciones de complicaciones como una flebitis superficial.

\section{Neuropatía venosa}

Constituye síntomas diversos consistentes en sensaciones de presión, opresión, quemazón,

pinchazos, picadura, desgarro, palpitaciones o escalofríos que se exacerban de forma importante en las menstruaciones y embarazo. Su origen está relacionado con el estado inflamatorio de la enfermedad. 


\section{Recanalización de la vena safena, factores de riesgo y tratamiento quirúrgico}

Vol. 3, núm. 1., (2019)

Piedad Shirley Barragán Villafuerte; Freddy Andrés Flores Vega; María Auxiliadora Cedeño Cevallos; Lucia Del Carmen Maza Suarez

Pesadez

Afecta sobre todo a las piernas. Aparece tras bipedestación prolongada. Suele ser deintensidad gradual, vespertina y disminuye con el reposo con el miembro elevado y ejercicios de relajación de la musculatura de los miembros.

\section{Calambres}

Suelen aparecer de noche o ligados al esfuerzo y al calor. Afectan a los músculos dela pierna, sobre todo pantorrilla y pie. Suelen despertar a los sujetos obligándolosa levantarse para masajearse la pierna. No son patognomónicos de enfermedad venosa.

\section{Varices}

Son venas dilatadas y tortuosas que reciben varios nombres según sus características y localización

- Telangiectasias: vénulas intradérmicas o subepidérmicas dilatadas, de menos de $1 \mathrm{~mm}$ de calibre, que tienden a confluir. También conocidas como arañas vasculares o capilares.

○ Reticulares: venas subdérmicas localizadas en la dermis reticular, tortuosas, de color azulado, con un calibre entre 1 y menos de $3 \mathrm{~mm}$.

○ Venas varicosas o tronculares: son venas subcutáneas dilatadas de 3 o más mm de diámetro, a menudo tortuosas. 


\section{Recanalización de la vena safena, factores de riesgo y tratamiento quirúrgico}

Vol. 3, núm. 1., (2019)

Piedad Shirley Barragán Villafuerte; Freddy Andrés Flores Vega; María Auxiliadora Cedeño Cevallos; Lucia Del Carmen Maza Suarez

- Corona flebectásica: describe un patrón en abanico de numerosas pequeñas venas intradérmicas sobre cara medial o lateral del tobillo. Constituye un signo temprano de EVC avanzada.

\section{Edema}

Es el aumento de líquido en el espacio intersticial como consecuencia del acrecentamiento de lapresión venosa capilar que lleva a la fuga de fluido desde el espacio intravascular. Clínicamente, se traduce como aumento del volumen de la pierna que al ser palpado produce signo de la fóvea positivo. Aparece en bipedestación, clinostatismo prolongado o con el calor. Es reversible o disminuye con la elevación del miembro, la contención elástica o el tratamiento farmacológico.Generalmente es unilateral y se agrava durante el día.

\section{Eccema}

Representa el eritema y descamación de la piel que afecta en un principio a la cara ínfero internade la pierna, para posteriormente progresar y comprometer toda la extremidad. Amenudo se localiza cerca de venas varicosas.

- Pigmentación: también llamada dermatitis ocre. Se produce cuando hay un oscurecimiento pardusco de la piel debido a hematíes extravasados, que suelen localizarse en eltobillo, pero puede extenderse hacia el pie o la pierna.

- Atrofia blanca: placas estrelladas, lisas, blanco marfil de consistencia esclerótica, salpicadas por telangiectasias y petequias rodeadas de un halo hiper pigmentado. Se localizan principalmente en el tercio inferior de piernas y pies. 


\section{Recanalización de la vena safena, factores de riesgo y tratamiento quirúrgico}

Vol. 3, núm. 1., (2019)

Piedad Shirley Barragán Villafuerte; Freddy Andrés Flores Vega; María Auxiliadora Cedeño Cevallos; Lucia Del Carmen Maza Suarez

- Lipodermatoesclerosis: también llamado hipodermitis, es una inflamación crónica localizada con fibrosis de la piel y tejido subcutáneo asociada con aumento del grosor del tejido dérmico y subdérmico. Suele ir precedido por edema difuso inflamatorio de la piel que puede llegar a ser doloroso. Clínicamente se aprecia dermatitis ocre en el tercio distal de las piernas, con endurecimiento de la piel, la cual se encuentra adherida a planos profundos. Es un signo de EVC avanzada.

- Úlcera venosa: es una solución de continuidad de la piel, sin tendencia a la cicatrización espontánea. Se localiza en las regiones perimaleolares, es de tamaño y profundidad variable pudiendo afectar a toda la circunferencia de las piernas y llegar a comprometer hasta el músculo. Es de fondo sucio, con fibrina y áreas de tejido de granulación.(Zancs, 2016). 


\section{Recanalización de la vena safena, factores de riesgo y tratamiento quirúrgico}

Vol. 3, núm. 1., (2019)

Piedad Shirley Barragán Villafuerte; Freddy Andrés Flores Vega; María Auxiliadora Cedeño Cevallos; Lucia Del Carmen Maza Suarez

\section{Figura $N^{\circ}$ 3. Trastornos tróficos en la insuficiencia venosa crónica: Hiperpigmentación ocre}

(1); Eccema (2); Úlcera venosa (3), Lipodermatoesclerosis (4); Úlcera cicatrizada (5); Atrofia

\section{blanca (6)}

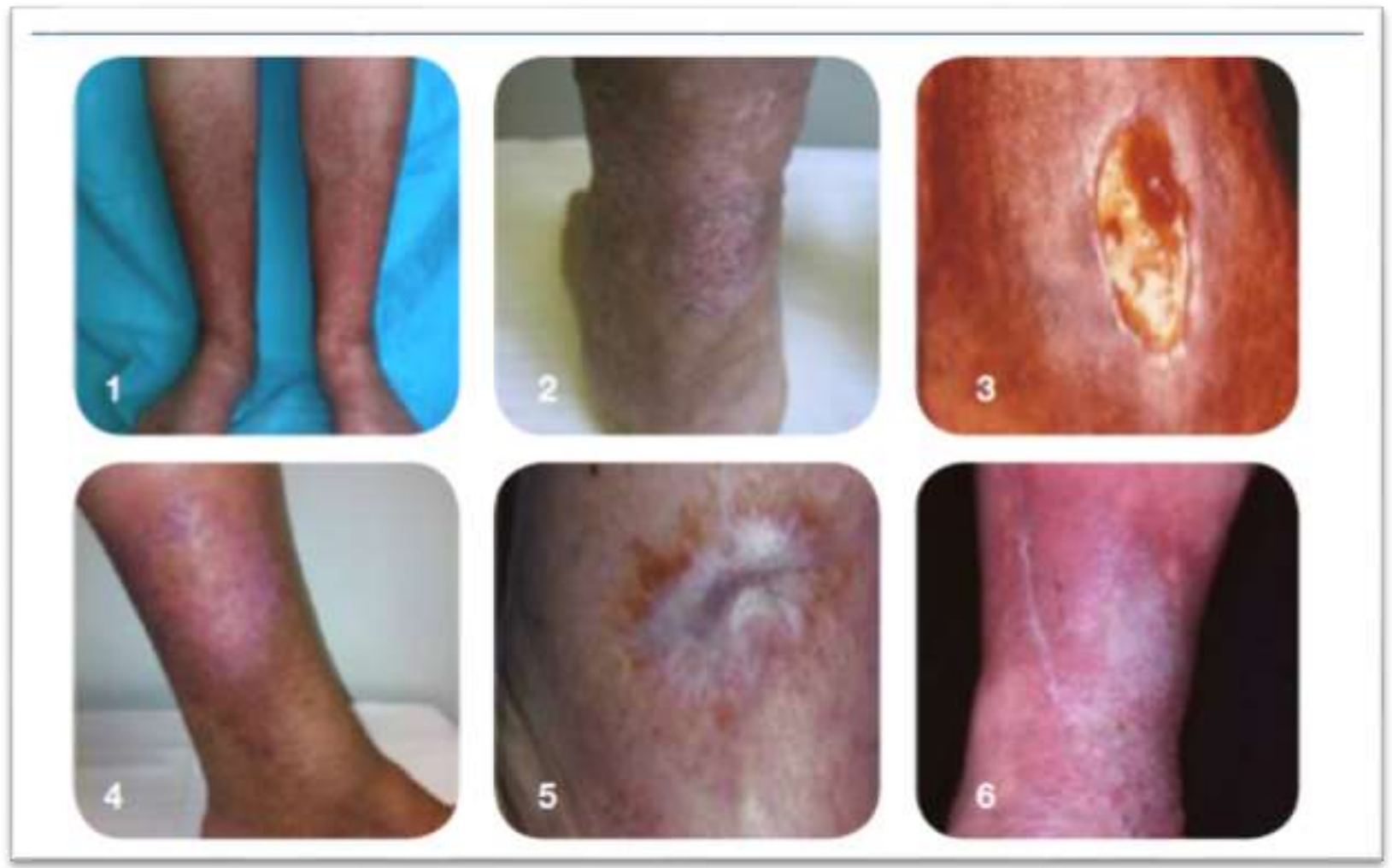

Fuente: Zans (2015)

El reflujo, a través del sistema venoso superficial y de las perforantes constituye uno de los elementos fisiopatológicos fundamentales en el desarrollo de la EVC, de ahí el importante desarrollo que han experimentado las técnicas correctoras microinvasivas en los últimos años, basadas en la eliminación mediante mecanismos físicos, mecánicos, químicos o quirúrgicos de los segmentos insuficientes. No obstante, sus indicaciones y su realización quedan fuera del ámbito de la AP. 


\section{Recanalización de la vena safena, factores de riesgo y tratamiento quirúrgico}

Vol. 3, núm. 1., (2019)

Piedad Shirley Barragán Villafuerte; Freddy Andrés Flores Vega; María Auxiliadora Cedeño Cevallos; Lucia Del Carmen Maza Suarez

El reflujo en el sistema venoso profundo es menos frecuente que el del sistema superficial, ya que está relacionado en la gran mayoría de las ocasiones con la enfermedad tromboembólica venosa, aunque también hay casos de insuficiencia venosa primaria. Su tratamiento siempre es quirúrgico y se basa en la reparación valvular, con una mayor tasa de éxito de las válvuloplastias en las insuficiencias de origen primario.

La obstrucción completa de las venas del sistema profundo es la responsable de los síntomas en un tercio de los síndromes posttrombóticos. En la mitad de los casos, se asocia a reflujo, provocando un mayor grado de hipertensión venosa y una peor evolución clínica. De igual manera, las obstrucciones proximales ilio-femorales tienen una peor evolución que las distales fémoro-poplíteas. La corrección de la obstrucción venosa mediante venoplastia y colocación de stent ha demostrado ser una terapia eficaz en la mayoría de los pacientes, incluso en presencia de reflujo.

Dada su peor evolución y la posibilidad de indicación quirúrgica, es importante tener en cuenta la posible presencia de una obstrucción venosa ilio-femoral en pacientes con claudicación venosa durante el ejercicio o con dolor desproporcionado.

\section{Tratamiento quirúrgico de la EVC}

La cirugía puede ser considerada en grandes edemas sintomáticos o en úlceras que nose controlen con terapia compresiva, si la obstrucción está por encima de la ingle. En estos casos, las obstrucciones crónicas de las venas iliacas o la cava inferior se tratan con recanalización y colocación de un stent. La cirugía del sistema venoso profundo para tratar el reflujo sigue siendo controvertida. 


\section{Recanalización de la vena safena, factores de riesgo y tratamiento quirúrgico}

Vol. 3, núm. 1., (2019)

Piedad Shirley Barragán Villafuerte; Freddy Andrés Flores Vega; María Auxiliadora Cedeño Cevallos; Lucia Del Carmen Maza Suarez

En los pacientes con úlceras recidivantes a pesar de un correcto tratamiento conservador, la cirugía del reflujo u obstrucción del sistema profundo puede ser considerada, debiendo realizarse en unidades especializadas con personal altamente entrenado.El objetivo de la cirugía, ya sea abierta, endovenosa o mediante escleroterapia, pretende eliminar el reflujo para restablecer la normalidad hemodinámica y extirpar las varices visibles para eliminar los síntomas, prevenir la recurrencia y minimizar las complicaciones.

Las técnicas mínimamente invasivas, menos cruentas, producen menor morbilidad quirúrgica, pero técnicamente son más complejas y presentan la misma tasa de recurrencia a largo plazo. Se recomienda individualizar los casos en función de la situación clínica, las disponibilidades técnicas y las preferencias del paciente. Las indicaciones de las distintas modalidades de tratamiento referidas son determinadas por la presencia de reflujo en safena y existencia de dilataciones varicosas, disponibilidad de los distintos métodos, experiencia del centro y preferencia del paciente.

- La eliminación del reflujo de safena, cuando existe, es el paso inicial, preferiblemente por técnicas endoluminales, seguido de cirugía con la excepción de las recurrencias varicosas debidas a crosectomía deficiente donde la escleroterapia es considerada una alternativa a la cirugía.

- Cualquier vena varicosa coexistente puede tratarse con extirpación de forma concomitante a la cirugía de safena o en un segundo tiempo si se ha efectuado termo ablación de la misma que pueda hacer prever una disminución de las dilataciones tributarias. Los pacientes deben ser informados de que la escleroterapia tiene una 


\section{Recanalización de la vena safena, factores de riesgo y tratamiento quirúrgico}

Vol. 3, núm. 1., (2019)

Piedad Shirley Barragán Villafuerte; Freddy Andrés Flores Vega; María Auxiliadora Cedeño Cevallos; Lucia Del Carmen Maza Suarez

elevada recurrencia de varices que puede tratarse asimismo con nueva escleroterapia incluyendo los ejes safenos.

- En varices aisladas (por ejemplo, si no existe insuficiencia de safenas), se emplean la exéresis o la escleroterapia. Deben considerarse las contraindicaciones potenciales de cada método como la pigmentación, que deben ser discutidas con el propio paciente.(Lavieri, 2015).

Tabla $N^{\circ}$ 4. Técnicas de repermeabilización quirúrgica del sistema venoso profundo

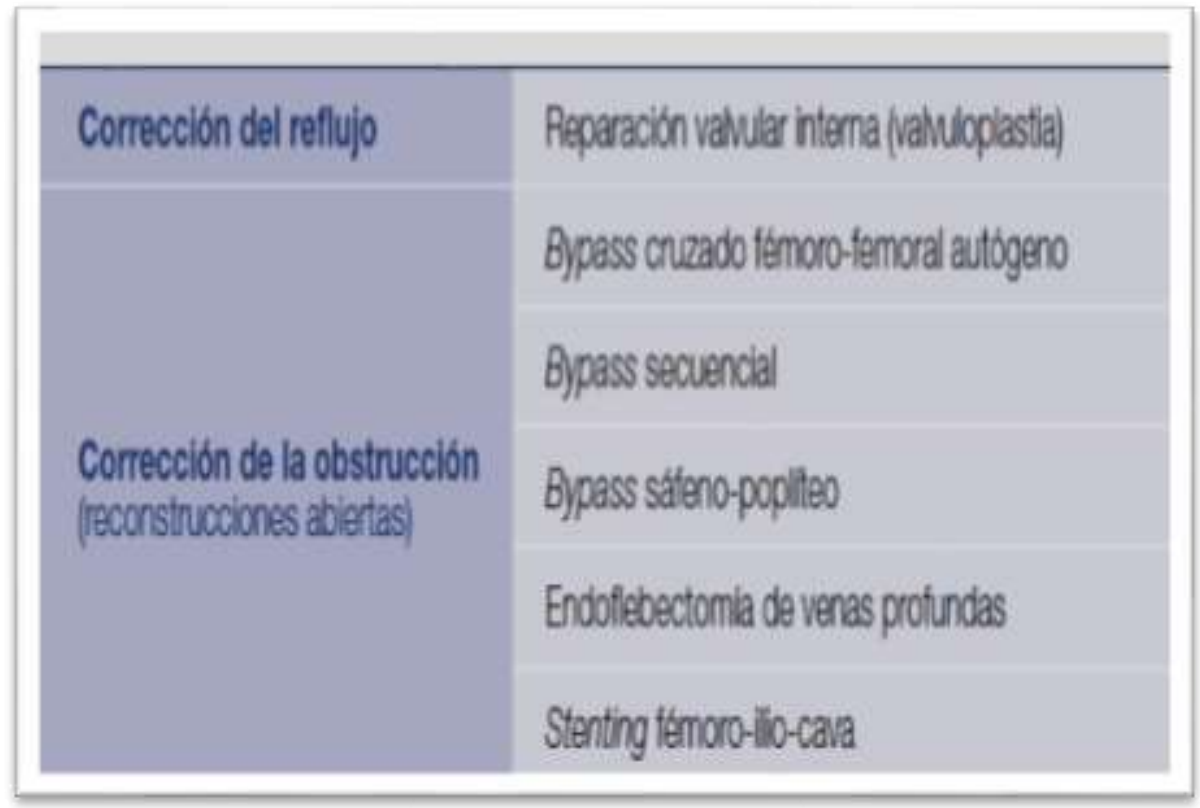

Fuente: Lavieri (2015)

\section{Conclusiones.}

La EVC es una enfermedad de curso crónico y progresivo que presenta un pronósticovital benigno, pero que por su frecuencia y elevada morbilidad, constituye un problema sanitario que 


\section{Recanalización de la vena safena, factores de riesgo y tratamiento quirúrgico}

Vol. 3, núm. 1., (2019)

Piedad Shirley Barragán Villafuerte; Freddy Andrés Flores Vega; María Auxiliadora Cedeño Cevallos; Lucia Del Carmen Maza Suarez

genera un enorme número de consultas tanto en AP como en los servicios de Angiología, donde el proceso de recanalización del trombo es de suma importancia; en el mismo se produce la destrucción del endotelio valvular de los segmentos implicados, de forma que la hipertensión venosa es desarrollada en relación al reflujo provocado, solo o combinado con un mayor o menor grado de obstrucción del drenaje venoso.

La prevención del síndrome posttrombótico se basa en la correcta dosificación y duración del tratamiento anticoagulante tras una trombosis venosa profunda, junto con la movilización precoz y la inmediata compresión elástica del miembro afecto. En pacientes con trombosis proximal, las medias de compresión por debajo de rodilla por un periodo de al menos dos años están recomendadas junto al tratamiento anticoagulante.

En la recanalización e insuficiencia valvular: lo normal es que la trombosis venosa profunda (TVP) se recanalice, pero lesione las válvulas de las venas profundas y las deje incompetentes, entonces el paciente presentará una hipertensión venosa crónica de la extremidad durante la bipedestación, que incluso se incrementa durante el ejercicio. Precisamente este es uno de los problemas cruciales del síndrome, es decir, que durante la deambulación no se reduzcan las presiones venosas por la bomba musculo-venosa de la pierna, que normalmente es muy efectiva.

Estas presiones venosas elevadas (tanto de pie como al caminar) se trasmiten a través de las venas perforantes desde sistema venoso profundo (SVP) a sistema venoso superficial (SVS). Clínicamente también pueden aparecer varices por la insuficiencia de las perforantes, sin 


\section{Recanalización de la vena safena, factores de riesgo y tratamiento quirúrgico}

Vol. 3, núm. 1., (2019)

Piedad Shirley Barragán Villafuerte; Freddy Andrés Flores Vega; María Auxiliadora Cedeño Cevallos; Lucia Del Carmen Maza Suarez

embargo es importante saber que en esta situación dichas varices no actúan como vía colateral de drenaje.

Como resultado de la lesión permanente en el sistema venoso profundo, bien por oclusión o por destrucción valvular, las venas perforantes del tobillo se dilatan y terminan haciéndose incompetentes dando lugar a hipertensión venosa anormal en la piel y tejido subcutáneo de la zona. La hipertensión venosa da lugar a hipertensión capilar con estasis circulatoria, resultando posteriormente los trastornos tróficos propios del síndrome. Las alteraciones cutáneas de estasis se producen por un escape de fibrina de los capilares. También se produce una lipoesclerosis y una dificultad de la difusión de oxigeno en los tejidos. El resultado final es la necrosis tisular y la ulceración cutánea.

El tratamiento quirúrgico, hasta hace poco era aceptado como el método terapéutico más s eficaz y de mejor pronóstico (actualmente ha sido sustituida por otras técnicas menos invasivas). Sus objetivos son: conseguir la desaparición de las propias venas varicosas con su repercusión clínica y estética y la profilaxis o el tratamiento de las complicaciones del síndrome varicoso. Este tratamiento es aplicado con distintos criterios, no solo en cuanto a indicaciones clínicas, sino también en lo referente a la radicalidad de la actuación.

A la técnica quirúrgica más radical han seguido oponiéndose los partidarios de una actuación más sencilla y limitada, que pretende conseguir suficientes resultados practicando cirugía ambulatoria basada en las interrupciones venosas con base hemodinámica: Este criterio no cubre el objetivo de erradicación de las venas varicosas. La técnica defendida por los que anteponen un criterio estético, efectuando varicectomía por incisiones mínimas (técnica de 


\section{Recanalización de la vena safena, factores de riesgo y tratamiento quirúrgico}

Vol. 3, núm. 1., (2019)

Piedad Shirley Barragán Villafuerte; Freddy Andrés Flores Vega; María Auxiliadora Cedeño Cevallos; Lucia Del Carmen Maza Suarez

Müller) no es aplicable a las varices de gran volumen y a las lesiones tróficas cutáneas. En el síndrome varicoso posttrombótico la actuación quirúrgica sobre las venas perforantes y el sistema venoso superficial (insuficientes secundariamente) es superponible al síndrome varicoso esencial.

\section{Bibliografía.}

Angiología, R. M. (2018). La Vena Safena. Salud Integral de la Universidad Autónoma de México, 22.

Arias, F. (2010). Paradigmas de la Investigación Científica. España: Editorial: Luces.

Blom, P. (2016). Vena Safena, Factores de Riesgo. Vida y Salud de la Universidad de la Habana en Cuba, 13.

Colling, H. (2016). Enfermedad Venosa Crónica ECV. La Habana: Editorial Científico Técnica. , 14.

Dávila, N. (2012). Paradigmas de la Investigación Científica. Pereire, Colombia: Editorial: Las Brisas.

Génova, V. (2016). Insuficiencia Venosa Crónica. Revista Médica de Mexicana Familiar ,, 14.

Lavieri, M. (2015). Técnicas Quirúrgicas en Cirugías Venosas. España: Editorial Oral.

Palett, R. (2017). Venas Varicosas. Tratamiento Quirúrgico. Boletín Informativo Médico de IMBIOMED, 12 .

Queralt, C. (2017). Recanalización de la Vena Safena. Revista de Anatomía Venosa de España., 11.

Quetz, C. (2016). Enfermedades Venosas Crónicas. Publicaciones Médicas de España, 27.

Vayaretti, O. (2016). Vena Safena, Tratamiento Quirúrgico y sus implicaciones Médicas. Revista Salud de la Escuela de Medicina de la Universidad de Los Andes ULA, Venezuela., 11.

Zancs, E. (2016). Recanalización de la Vena Safena. Chile : Editorial Eace. 